\section{GUÍA DE SELECCIÓN DE ERP EN LAS PEQUEÑAS Y MEDIANAS EMPRESAS MEXICANAS}

\section{GUIDE TO THE SELECTION OF ERP IN MEXICAN SMALL AND MEDIUM-SIZED ENTERPRISES}

\author{
Igor Rivera \\ Instituto Politécnico Nacional - UPIICSA
}

iariverag@ipn.mx

María del Rosario Pérez Salazar

Instituto Tecnológico Superior de Tantoyuca rosario.perez.salazar@gmail.com

\footnotetext{
* Agradecemos el apoyo otorgado para el desarrollo de este artículo al Instituto Politécnico Nacional, Instituto Tecnológico de Tantoyuca y Secretaría de Ciencia, Tecnología e Innovación del D.F. México (ICYTDF/325/2011)
}

Cómo citar este artículo/ Citation: Rivera, I. y Pérez Salazar, M. R. (2013). Guía de selección de ERP en las pequeñas y medianas empresas mexicanas. Arbor, 189(760):a025. doi: http://dx.doi. org/10.3989/arbor.2013.760n2011

Recibido: 4 mayo 2010; Aceptado: 18 marzo 2011.

RESUMEN: Los Enterprise Resource Planning (ERP) son Sistemas de Información caracterizados por tener una base de datos común para todos los departamentos de la empresa y son utilizados por las organizaciones para administrar sus datos con el fin de tomar mejores decisiones. Sin embargo, estudios recientes demuestran que el $92 \%$ de los proyectos ERP no terminan con éxito (De Pablos Heredero y De Pablos Heredero, 2009). El objetivo de este artículo es diseñar una guía de selección de ERP en las Pymes mexicanas. En primera instancia se realizó un examen de la literatura científica, el cual se dividió en: propuestas metodológicas, casos de estudio, estudios microsociológicos y factores críticos de éxito para proyectos ERP. Para complementar nuestro estudio teórico se entrevistaron consultores, utilizadores y expertos en proyectos ERP, los cuales apoyaron nuestra concepción de esta problemática en el ámbito local. Finalmente se diseñó la guía de selección de ERP.
Copyright: (C) 2013 CSIC. Este es un artículo de acceso abierto distribuido bajo los términos de la licencia Creative Commons Attribution-Non Commercial (by-nc) Spain 3.0.

ABSTRACT: Enterprise Resource Planning (ERP) systems are information system characterised by having a shared database for all the company's departments. Organisations use ERP systems to manage their data and help improve decision-making. However, recent studies show that $92 \%$ of ERP projects do not end in success (De Pablos Heredero and De Pablos Heredero, 2009). This paper aims to design a guide to ERP selection for Mexican SMEs. It starts with a review of the scientific literature, which is divided into methodological proposals, case studies, micro-sociological studies, and critical success factors for ERP projects. To complement our theoretical study we interviewed consultants, users and ERP project experts, who supported our conception of this issue at local level. Finally, we designed an ERP selection guide. 


\section{INTRODUCCIÓN}

Las empresas son organizaciones que cuentan con una gran cantidad de datos que no siempre son aprovechados para ser más productivas, competitivas y rentables. Los sistemas de información actuales han sido capaces de apoyar de manera importante la gestión y el control de estos datos empresariales. Uno de estos sistemas han sido los Enterprise Resource Planning (ERP). Los ERP son herramientas informáticas que utilizan las organizaciones para gestionar de mejor manera sus datos y por lo tanto sus recursos. Estos sistemas se caracterizan por contar con una base de datos común para todos o la mayor parte de los departamentos de la organización, lo cual ayudará a automatizar y sincronizar las transacciones de las diferentes actividades de la empresa. Un ERP generalmente está compuesto por múltiples módulos (Esteves y Pastor, 1999; Baptiste et al, 2001).

A lo largo de los últimos años se observa una tendencia creciente en el entorno empresarial hacia el desarrollo y difusión de los sistemas ERP (Sheu, Chae y Yang, 2004). Mientras que la mayoría de las empresas grandes ya han invertido en un sistema ERP, la difusión de este tipo de sistemas de información se está extendiendo a las medianas y pequeñas empresas (Pymes) (Forger, 2000). Sin embargo, estudios recientes han demostrado que el $92 \%$ de los proyectos ERP no terminan con éxito (De Pablos Heredero y De Pablos Heredero, 2009). Una razón de estos fracasos corresponden a la falta de una adecuada selección de la herramienta (Rivera, 2005 y Teltumbde, 2000). Es por eso que consideramos que estudiar la etapa de selección del ERP es de gran importancia para el seguimiento del proyecto. Así, el objetivo de este artículo es el de diseñar una guía de selección de Enterprise Resource Planning en las Pymes mexicanas.

Las fases del ciclo de vida de un proyecto ERP consisten en 1) planeación del proyecto y toma de decisión de realizarlo, 2) selección de la herramienta, del editor y del implantador o consultor, 3) implantación del ERP y 4) puesta en marcha, 5) uso, 6) evolución y 7) Fine-tunning. Enseguida mencionaremos las dos primeras fases, las cuales se consideran en este artículo: En la etapa de planeación del proyecto se define si un sistema ERP es la solución adecuada para la empresa en vez de otro tipo de sistema. Se debe cuestionar la necesidad de un ERP, sus objetivos y beneficios, así como el análisis del impacto que su adopción tendría a nivel operacional y organizacional. Si se ha decidido adquirir un ERP, la empresa puede continuar con la etapa de selección. Esta etapa inicia con la selección de los integrantes del equipo de proyecto, hasta la firma del contrato del ERP, de la empresa editora (organización que se encarga de desarrollar el ERP) y del equipo integrador (empresa consultora que ayuda a implantar el ERP).

\section{METODOLOGÍA DE INVESTIGACIÓN}

La metodología que se llevó a cabo en esta investigación ha sido, en una primera instancia de tipo teórica, para continuar con entrevistas a expertos en el tema y finalizar con una síntesis sobre nuestras investigaciones. Se realizó una revisión de la literatura académica referente a la fase de selección de los sistemas ERP. Para llevar a cabo este estudio nos basamos en artículos científicos, utilizando como fuente de consulta diferentes bases de datos. Para complementar nuestro estudio, nos basamos en libros de investigación de editoriales de reconocido prestigio, así como de tesis doctorales. Aunque existe una cantidad importante de artículos relacionada a los ERP, pocos son los artículos que se interesan a la selección de ERP. En la selección de la documentación referente a nuestro objetivo de análisis, el número de publicaciones considerada fue de 39 títulos, de los cuales 31 son artículos científicos, 4 libros de investigación (conformados por varios capítulos de investigación), 2 tesis de maestría y 2 tesis doctorales. Algunos de los artículos científicos analizados no se encuentran en los análisis presentados en este escrito, debido a que sus aportaciones ya están consideradas en los artículos presentados. En un estudio sobre la literatura de ERP en 2001, Esteves y Pastor (2001) hacen mención de solo 11 artículos científicos relacionados con la selección de ERP. El siguiente paso fue llevar a cabo un examen de la literatura, por lo cual dividimos los materiales científicos en cuatro apartados: propuestas metodológicas, casos de estudio, estudios microsociológicos y factores críticos de éxito en los proyectos ERP. Una vez efectuada esta categorización se realizó el análisis de cada una de las partes. El siguiente paso en la elaboración de la guía de selección, fue seleccionar del apartado de propuestas metodológicas a la metodología para la selección de sistemas ERP de Chiesa (2004) como base de nuestra guía, ya que consideramos que esta propuesta metodológica señala actividades muy puntuales dentro del proceso de selección de los sistemas ERP. Además de la revisión de la literatura, para la construcción de la guía se realizaron y analizaron 5 entrevistas a especialistas en los proyectos ERP: consultores, vendedores, utilizadores y líderes de proyecto.

\section{EXAMEN TEÓRICO}

Lo que se presenta en este apartado es una selección de los principales artículos, referentes a la selección de ERP, revisados en revistas científicas, tesis doctorales y libros de investigación. Después de haber analizado la literatura y debido a la naturaleza de cada tipo de publicaciones decidimos dividirla en cuatro grandes rubros: propuestas metodológicas, casos de estudio, estudios microsociológicos y factores críticos de éxito para proyectos ERP. 
Las propuestas metodológicas revisadas se basan en realizar el proceso de selección de acuerdo a que tanto los ERP se adecuan a los requisitos de la organización. A continuación se presenta cada una de estas metodologías:

a) La metodología SHERPA (Systematic Help for an ERP Acquisition) va desde la búsqueda del candidato hasta la firma del contrato y sus fases son: fase 0.1: estudiar la estrategia y los procesos de negocio; fase 0.2: decidir adoptar o no un ERP; fase 1: búsqueda de candidatos y efectuar primer filtro; fase 2: analizar en detalle los candidatos y efectuar segundo filtro; fase 3: análisis y demostración de candidatos, y visitas a proveedores; y fase 4: decisión final, negociación y planificación(Estay Niculcar y Pastor, 2003).

b) La metodología para la Selección de Sistemas ERP de Chiesa (2004) se organiza en tres fases las cuales se dividen en actividades: fase 1: selección del ERP (a) documentar necesidades, análisis de necesidad, determinar equipo de proyecto; b) primera selección, buscando en el mercado de ERP, primer contacto con proveedores, entrevistar posibles candidatos y recopilar información, armado de listado de criterios a tener en cuenta, evaluar los candidatos, documentación de la selección y armado del plan de trabajo; c) selección final, organizar visitas a los proveedores, demostración del producto, decisión final y negociación con el proveedor ERP); fase 2: selección del equipo de consultoría (a) documentar bases de la búsqueda, organizar la búsqueda, armar listado de criterios para seleccionar consultora; b) selección de candidatos, entrevistar posibles candidatos y recopilar información, evaluar los candidatos, decisión final y negociación) y fase 3: presentación y planificación general del proyecto.

c) El método propuesto por Wei, Chien y Wang (2005) para el proceso de selección de ERP se basa en el método del proceso analítico jerárquico (AHP) y se presenta en una serie de pasos: paso 1: formar un equipo de proyecto y colectar toda la información posible acerca de los sistemas ERP y de los proveedores; paso 2: identificar las características del sistema ERP; paso 3: construir una estructura de objetivos para desarrollar la jerarquía de los objetivos fundamentales y la red de medios para conseguir esos objetivos; paso 4: extraer los atributos para la evaluación de los sistemas ERP de la estructura de los objetivos; paso 5: filtrar los vendedores no calificados, realizando preguntas específicas, que se formulan de acuerdo a los requisitos del sistema; paso 6: evaluar los sistemas ERP mediante el método AHP y paso 7: discutir los resultados y tomar la decisión final. d) El modelo MERPAP (Model of the ERP Acquisition Process), basado en el resultado de casos de estudio, indica seis distintos procesos iterativos: planificación (formación del equipo de adquisición, estrategias de adquisición, definición de requerimientos, establecimiento de criterios de selección y evaluación, cuestiones de adquisición, análisis del mercado), búsqueda de información, selección, evaluación, elección y negociaciones; se centra en la adquisición del ERP mediante un estudio de selección y evaluación de los proveedores y del sistema. Para esta evaluación los elementos a considerar son: a) proveedor, b) parte funcional, c) técnica (Verville, 2002).

e) Teltumbde (2000) presenta un marco para evaluar proyectos ERP, el cual se basa en la comprensión del mapa completo de las características de Ios ERP siguiendo 10 criterios propuestos. El marco comprende siete campos de acción: creación de la infraestructura organizacional, constitución del repertorio de productos de ERP, fase de preparación, fase de ajuste, fase de evaluación y selección, aprobación de la selección y evaluación de mitad de curso.

f) Stefanou (2000) propone un marco para el proceso de selección de ERP que consta de tres fases: fase 1: visión de negocio: considera a la visión del negocio como punto de partida para la adquisición del ERP; fase 2: requerimientos del negocio contra las limitaciones y el deseo de cambiar: consiste en el examen detallado y la definición de las necesidades del negocio, y de las diversas limitaciones; fase 3: selección y evaluación del ERP, consistente en la selección y la evaluación del proveedor correspondiente, de productos y servicios de apoyo para satisfacer las necesidades de negocio. Esta fase también incluye la estimación del costo de la inversión necesaria para la adquisición, implementación y mantenimiento del sistema propuesto a través de su ciclo de vida.

Al analizar las metodologías enlistadas tenemos que una parte importante de los artículos sugieren que es necesario seleccionar aquellas herramientas que contengan la mayor parte de los requisitos de la empresa, dentro de las funcionalidades que proporciona el ERP. Para la aplicación de la mayoría de las metodologías de selección se considera tomada la decisión de implementar un ERP y no otro tipo de sistema, a excepción de dos propuestas, la metodología SHERPA que considera dos etapas previas, la fase de estudiar la estrategia y los procesos de negocio y la fase de decidir adoptar o no un ERP, y la metodología para la selección de sistemas ERP, que dentro de la fase de selección incorpora las actividades de analizar y documentar la necesidad de un ERP. Asimismo, los mé- 
todos analizados advierten que para que los ERP sean implementados, la organización tuvo que haber llevado un trabajo de revisión de sus procesos, además de saber qué áreas estarán involucradas e impactadas por el cambio. Solo un modelo de los analizados, considera en su fase 1 a la visión de negocio como punto de partida para la adquisición de un ERP y el realizar un examen de las necesidades y limitaciones de negocio en la fase 2 . En este sentido, encontramos cuando analizamos la realidad de las respuestas, que algunas veces la decisión de adoptar un sistema ERP y no otra herramienta informática no está en el dominio de la misma empresa, sino le es impuesta a la empresa influencias externas como, la fusión con otra empresa que utiliza un ERP o por exigencia de un proveedor o un cliente importante para la empresa.

La segunda clasificación que llevamos a cabo en este artículo es referente a casos de estudio que hicieron los autores en una empresa. Aunque este estudio abarca varias etapas de un proyecto ERP, existen elementos rescatables de la etapa de selección. Los casos de estudio analizados son:

a) Estudio realizado en una empresa mediana en la cual inicia con un análisis completo de la empresa, tomando en cuenta los elementos importantes mediante el proceso PSI (Planificación de Sistemas de Información) y una vez concretados los objetivos a realizar para la implantación, se analizan varios ERP's existentes en el mercado y se estudian para determinar cuáles son los que mejor se adaptan a la empresa (García, 2008).

b) Expósito, Tomás y Vicedo (2004) plantean una metodológica para el análisis del sistema de información y propuesta de solución en una empresa textil pequeña. En primer lugar se realiza un estudio de las funciones y flujos informativos de la empresa mediante la metodología IDEFO (Integration Definition for Function Modeling). A continuación, se plantea un análisis del sistema de información actual de la empresa basado en Diagramas de Flujo de Datos, permitiendo identificar las carencias y limitaciones que posee el programa actual de gestión de la empresa, para así plantear posibles soluciones basadas en la implantación de un ERP.

c) El estudio de Sternad, Bobek, Dezelak y Lampret (2009) se trata de una investigación llevada a cabo en una empresa mediana, en donde se analizan dos retos que el equipo del proyecto debía enfrentar: a) la implementación de un sistema ERP que significa un nuevo concepto y modelo de proceso para la empresa y b) en las Pymes, los empleados tienden a cubrir múltiples roles y responsabilidades en la organización y en los procesos. La empresa hizo la selección del producto, un ERP comercial, el cual es considerado más caro que los productos locales y otros proveedores en términos de la licencia y servicios de implementación, pero ofrecía la funcionalidad probada que la empresa necesitaba.

Los tres casos de estudio revisados consideran proyectos ERP desarrollados en pequeñas y medianas empresas y rastrean la aplicación de una metodología de selección específica en el desarrollo de proyecto ERP. La metodología se utiliza (en los dos primeros casos) para realizar un análisis completo de la empresa. Basándose en los objetivos de cada estudio, se analizan los sistemas ERP existentes en el mercado o las diferentes variantes de los ERP (ERP generalistas, ERP específicos para un sector o ERP a la medida). Los dos primeros casos de estudios concluyen con la decisión acerca del ERP que mejor se adapta a los objetivos de la empresa, y por lo tanto que mejor satisface las necesidades de la empresa. El tercer caso apunta aportaciones importantes acerca de los retos que enfrentan las Pymes en el proceso de selección de un ERP.

Nuestra tercera clasificación en este escrito lleva por nombre "Estudios microsociológicos" y se trata de investigaciones de largo plazo, en donde los autores llevaron a cabo estudios muy puntuales de proyectos ERP en medianas empresas. Los estudios analizados son:

a) Rivera (2005) analiza el antes, durante y después de la selección de un ERP en una empresa mediana y propone que los elementos a considerar en la selección del ERP son: apoyo total de la dirección; conformación del equipo ERP con personal que puede tomar decisiones en sus respectivos departamentos; análisis de los procesos actuales y futuros que desea la empresa; short list con la ayuda de expertos y sin privilegiar algún $E R P$, editor o integrador; análisis técnicos y financieros a editores e integradores; visita a empresas que ya utilizan los ERP seleccionados; examen a los integradores, sobre una serie de preguntas relacionadas con los procesos actuales y futuros de la organización; selección tomando en cuenta el beneficio de la empresa y no de un solo departamento.

b) El segundo estudio (Urcelay, 2003) que analizamos sobre empresas manufactureras medianas que hayan implantado un sistema ERP en sus políticas de gestión, presenta una lista de criterios que puede servir de orientación en el momento de seleccionar un sistema de gestión integral: 1) criterios de funcionalidad, 2) criterios técnicos, 3) criterios de proveedor, 4) criterios de servicios ofrecidos, 5) criterios económicos. Plantea tres etapas a seguir en el proceso de selección de ERP: etapa 1: descartar aquellos sistemas que no cum- 
plan los criterios mínimos establecidos y exigidos; etapa 2: llevar a cabo contactos directos con los proveedores, midiendo el grado de cumplimiento de los criterios de una forma escrita, aumentando gradualmente el nivel de exigencia y adecuación de los proveedores y sus productos, hasta obtener una relación reducida de candidatos; etapa 3: definir aspectos tales como el plan de implantación, los cambios que implicará la adopción de cada paquete dentro de la empresa, el nivel de implicación que asumirá el proveedor en el mantenimiento y evolución del sistema o las condiciones de contrato demandadas por cada uno de ellos.

Los estudios microsociológicos revisados analizan los procesos sociotécnicos subyacentes que dan lugar a las relaciones entre los miembros de un equipo de proyecto ERP y los procesos de la empresa. En los dos casos son analizados conjuntos de empresas medianas. Los estudios microsociológicos, por su carácter situacional emplean principalmente métodos cualitativos como la etnografía, la investigación acción, las historias de vida y los relatos y están basados en observaciones sistemáticas y análisis de la vida cotidiana. Este tipo de estudios, si bien no son probados en varias empresas, tienen el beneficio de que son análisis puntuales y específicos que ayudan a entender las entrañas de lo que pasa en los proyectos ERP.

El último grupo de escritos analizados en este artículo, es el relacionado con los "factores críticos de éxito para proyectos ERP", los cuales señalan puntualmente factores que han sido probados y han dado beneficios al desarrollo de proyectos ERP de estudios teóricos en donde se analizan un importante número de artículos científicos, relacionados a la selección de ERP. Las publicaciones revisadas son:

a) Esteves y Pastor (2004) proponen un modelo de factores críticos de éxito (FCE) para proyectos $E R P$, en donde recopilan todos los FCE encontrados en artículos sobre ERP, determinan las similitudes o los patrones comunes entre ellos y los categoriza en: a) factores organizacionales estratégicos: apoyo continuo de la alta dirección, gestión efectiva del cambio organizacional, buena gestión del ámbito de proyecto, composición adecuada del equipo de proyecto, reingeniería de los procesos de negocio, papel adecuado del líder del proyecto, papel adecuado del gestor del proyecto, implicación y participación de los usuarios y confianza entre las partes afectadas; b) factores tecnológicos estratégicos: estrategia de implantación ERP adecuada, evitar desarrollos a la medida y versión adecuada del ERP; c) factores organizacionales tácticos: equipo y consultores ampliamente dedicados, comunicación decidida hacia dentro y hacia fuera, planificación formalizada del proyecto, programa de formación adecuado, anticipación preventiva de problemas, uso adecuado de consultores y responsables debidamente autorizados; y d) factores tecnológicos tácticos: conocimiento suficiente de los sistemas preexistentes, plan de pruebas formalizado y proceso de migración de datos adecuado.

b) Stefanou (2001) clasifica a los factores críticos de éxito en factores en el nivel estratégico y factores en el nivel operacional. Dentro del nivel estratégico se encuentra la contribución del ERP a la visión y estrategia de negocio, la coherencia de la estrategia de negocios y de tecnología, la flexibilidad y la adaptabilidad de la solución ERP a las condiciones cambiantes, impacto del ERP en la toma de decisiones, impacto del ERP en las redes empresariales de cooperación, estimación del costo total de propiedad del ERP y el impacto sobre los recursos de las organizaciones, etc. En el nivel operacional se toma en cuenta dos aspectos: a) impacto del ERP en: costos de transacción, tiempo para completar las transacciones, grado de integración de los procesos de negocio, intercambio de información organizacional, y b) estimación de los costos debido a: resistencia de los usuarios, la capacitación del personal, consultores externos, aplicaciones adicionales y el tiempo de inactividad del sistema.

c) Otro estudio interesante a analizar es el desarrollado por Sternad, Bobek, Dezelak y Lampret (2009), basándose en 24 documentos previamente publicados, que se centran en los factores críticos de éxito de selección de ERP: apoyo y participación de la dirección; metas claras, objetivos y ámbito de aplicación; organización y competencia del equipo del proyecto; gestión de proyecto; líder de proyecto; elección de la arquitectura (selección del paquete) y personalización mínima.

d) También de Sternad, Bobek, Dezelak y Lampret (2009), se analiza un estudio realizado en 45 Pymes para investigar la importancia de los FCE en la implantación de un ERP. Se mencionan las respuestas más frecuentes de las empresas dentro del proceso de selección: a) en relación a la lista corta de candidatos ( 2 a 4 ) en la búsqueda preliminar de aplicaciones ERP, las respuestas fueron: ERP de proveedores locales, ERP comerciales, apuntando a las soluciones de proveedores locales como las más económicas; b) respecto a las razones para elegir un determinado ERP las respuestas fueron: la integridad del ERP seleccionado, la eficiencia y la estabilidad de la operación del ERP, el apoyo del proveedor ERP, el costo y el precio del ERP, y la exigencia del 
propietario o de otros socios comerciales (clientes, proveedores, etc.).

Los FCE son un conjunto de recomendaciones para alcanzar un proyecto ERP exitoso. La mayoría de las publicaciones repasadas son revisiones de estudios realizados acerca de los factores críticos de éxito mayormente mencionados en trabajos previos. Las dos primeras publicaciones señalan una distinción de estos factores críticos de éxito al clasificarlos en dos niveles: factores estratégicos y factores tácticos u operacionales. Se destaca que los FCE más importantes en la etapa de selección de un ERP son el papel adecuado del gestor del proyecto, una gestión efectiva de cambio organizacional, el uso adecuado de consultores, la implicación del usuario, metas claras, objetivos y ámbito de aplicación, organización del equipo del proyecto y personalización mínima del paquete. Los autores analizados, sugieren darle más importancia a los factores organizacionales que a los tecnológicos, es decir, centrarse más en las personas y en el proceso que en la propia tecnología.

\section{ENTREVISTANDO A LOS EXPERTOS}

Como fue señalado en la metodología de investigación, una parte de nuestra investigación se basó en la realización, análisis y síntesis de entrevistas a consultores, utilizadores y expertos en la selección e implantación de ERP.

El entrevistado 1 tiene varios años de experiencia como vendedor y consultor de los principales sistemas ERP en México. El describe que en las Pymes, el proceso de selección involucra de 2 a 3 personas que son: director general, una persona del departamento de sistemas y el contador, además explica que la decisión de selección del ERP está basada principalmente en la experiencia de cada uno de estos actores. También indica que un error que comenten las empresas durante la etapa de selección es el no realizar un análisis de la necesidad de adquirir o no un ERP, ya que algunas empresas adquieren este sistema por: a) recomendación de un empresario, b) observar que este sistema funcionó en otra empresa, c) recomendación del encargado de sistemas o el contador. Por otro parte, en su papel como consultor, el entrevistado recomienda a las empresas realizar un estudio de factibilidad antes de considerar adquirir un ERP, ya que a su parecer, gracias a este análisis la empresa se dará cuenta de los problemas reales de la empresa, además identificará los procedimientos que el sistema soportaría y complementaria. Este entrevistado concluye que en el éxito de la implementación de un ERP, el 50\% está en la decisión del ERP seleccionado, y la parte restante tiene que ver con la manera en que la consultoría trabajará con la empresa.

La entrevistada 2 cuenta con 20 años de experiencia en el área de los ERP's, primero como líder de proyec- to en una implantación de un MRP, y después como consultora de procesos en proyectos de implantación de ERP. Ella afirma, dentro de su papel como consultor, que en los en los años 90 la selección de un ERP se hacía empíricamente y en estos últimos años, cuidando los gastos totales, el $90 \%$ de los clientes que han contratado los servicios de su equipo de consultoría, han realizado una selección de ERP formal. Dentro del proceso de selección de un ERP. Además, esta entrevistada recomienda los siguientes puntos: a) involucrar todas las áreas de la empresa, y satisfacer en la medida de lo posible las necesidades de cada área, debe estar involucrado todo el personal, b) el compromiso de la dirección es indispensable desde el arranque del proyecto, y sería ideal que el proyecto ERP se considerase como la prioridad número dos durante el tiempo programado, siendo la operación de la empresa la prioridad uno, c) acordar desde un principio con el proveedor ERP si el sistema funcionará con los impuestos que establece Hacienda y d) asegurar que el sistema será útil para el proceso y para el ambiente de trabajo de la empresa (manufactura, comercializadora, servicios).

El entrevistado 3 tiene una experiencia de 10 años como consultor y utilizador de ERP. El describe que el proceso de selección de ERP en las Pymes comienza con una búsqueda en internet realizada por el personal de sistemas, resultando en una lista de paquetes, cuyas especificaciones son referenciadas a los directores, que a su vez preguntan a la persona a cargo del área en específico donde se implantará; y es el director el que toma la decisión final en base al costo. También recomienda que durante la etapa de selección de ERP, los mandos medios y superiores de las organizaciones deben reunirse para exponer sus problemáticas, una vez identificadas las problemáticas en los procesos, especialmente en el control de la información, modelos de planeación, modelos de inventarios, modelos de reposición y compra etc., entonces se debe definir un grupo de personas que se dediquen a buscar que opciones existen en el mercado. Más tarde el entrevistado comenta que de esta búsqueda resultará una lista de todos los ERP disponibles junto con sus habilidades estándar y seguirá el realizar un análisis costo beneficio y hacer una diferenciación entre los sistemas ERP para elegir el tipo de aplicación para la empresa.

El entrevistado 4 es integrante del equipo de proyecto en una implantación de ERP, además de contar con 16 años de experiencia como consultor en diversos despachos de Sistemas de Información. El considera esencial dentro del proceso de selección de ERP que las empresas: a) realicen un mapeo de procesos por áreas de negocio, compras, ventas, inventarios, finanzas para identificar los procesos que soportará el ERP, b) conozcan que es un ERP y sus funciones, c) 
cuenten con un consultor de negocios que les ayude a hacer una evaluación de ese tipo de proyectos.

El entrevistado 5 cuenta con 17 años de experiencia en Administración de la Cadena de Suministro y Sistemas de Información. El comenta que en la actualidad las Pymes no realizan un proceso formal de selección de ERP, sino que lo hacen por recomendación, por búsqueda en un directorio o comparando presentaciones de las opciones que encuentran. A su vez recomienda contratar los servicios de un consultor para tomar la decisión de adquirir un ERP, señalando que aun cuando sea un proceso más largo, esto asegura un mayor éxito en la posterior implantación del ERP.

\section{GUÍA DE SELECCIÓN DE UN ERP}

En este apartado se desarrolla la guía de selección de un ERP para Pymes mexicanas. Dentro de las fases de un proyecto ERP, se considera a la planeación como la primera fase de este tipo de proyectos, por lo que no podemos hablar de la etapa de selección sin hablar de la planeación de un proyecto ERP.

Basándonos en dos metodológicas, la primera realizada por Stefanou (2000) y la segunda por Estay Niculcar y Pastor (2003), en la etapa de planeación se debe considerar a la visión del negocio como punto de partida para la adquisición del ERP. En esta fase del proyecto ERP, se debe estudiar la estrategia y los procesos de negocio, además se deben definir las necesidades del negocio y las diversas limitaciones, todo esto para justificar la decisión de adoptar o no un sistema ERP. En este sentido, compartimos la posición de una de nuestros entrevistados, que en su rol como consultor menciona que en esta etapa del proyecto ERP se debe conocer, comprender y corregir la parte administrativa, de información y de las operaciones de la empresa, para así decidir si la empresa realmente necesita un sistema ERP o un paquete informático de diferente confección.

Como se mencionó en la metodología tomaremos como base de esta guía a la propuesta metodológica de Chiesa (2004). Esta metodología la estaremos complementando en la primera fase con la aportación de un estudio microsociológico (Rivera, 2005). Así, la guía que proponemos está dividida en tres fases: selección del ERP/editor, selección del equipo integrador (empresa consultora que ayuda a implantar el ERP) y presentación y planificación general del proyecto. Cada una de las fases que estaremos describiendo contiene una serie de actividades.

Fase 1. Selección del ERP/editor

Actividad 1. Determinar el equipo de proyecto: Antes de comenzar la búsqueda del ERP se debe nombrar a los responsables del proyecto, además de que es importante que el proyecto tenga el apoyo total de la dirección (estos dos elementos son igualmente propuestas por Esteves y Pastor, 2004 y Sternad, Bobek, Dezelak y Lampret, 2009 y nuestros entrevistados). Se sugiere la conformación del equipo ERP con personal que pueda tomar decisiones en sus respectivos departamentos, un equipo con una visión estratégica, en el cual, por lo menos deberá existir un actor conocedor de los sistemas ERP y sus características.

Actividad 2. Analizar y documentar necesidades: Se deberá realizar un análisis de los procesos actuales de la empresa, así como de los procesos que se pretende mejorar con la adopción del ERP. Lo anterior, como base para definir claramente una necesidad que podría ser satisfecha mediante un producto ERP. Uno de nuestros entrevistados sugiere que en esta etapa se realice un mapeo de procesos por áreas de negocio. Esta actividad se deberá considerar, como lo menciona Segrestin (2004), una oportunidad para innovar integralmente los procesos de negocio de la empresa. En esta actividad compartimos la posición de nuestro entrevistado 1 cuando recomienda que antes de adquirir un sistema ERP, las empresas deben de realizar un análisis para identificar los procedimientos que soporta y complementa el sistema. Para el mismo entrevistado, en la actualidad, las Pymes mexicanas no realizan un análisis de la necesidad de adquirir o no un ERP.

Actividad 3. Armar un listado de criterios a evaluar: Desarrollar una lista de criterios que servirá como base para la evaluación de los ERP. En esta actividad, la comunicación entre los diferentes departamentos de la empresa sobre las actividades y requisitos a tomar en cuenta, facilitará el establecimiento de criterios que apuntarán al beneficio de la empresa y no de un solo departamento. Esto lo corroboramos con algunos de nuestros entrevistados, que cuentan con una visión de las Pymes mexicanas. Para Urcelay (2003), en este listado se debe considerar 1) criterios de funcionalidad, 2) criterios técnicos, 3) criterios de proveedor, 4) criterios de servicios ofrecidos y 5) criterios económicos.

Actividad 4. Realizar la primera búsqueda en el mercado de ERP: Efectuar la búsqueda en el mercado de los ERP disponibles, establecer el primer contacto con los proveedores, llevar a cabo entrevistas a posibles candidatos y recopilar información. Esta actividad también incluye la evaluación de los candidatos, descartando aquellos sistemas que no cumplan con los criterios exigidos y documentar el grado de cumplimiento de cada uno de los ERP aceptados en esta fase. Cabe resaltar que en México no existe una lista crítica de los sistemas ERP que resalte las características y recomendaciones de cada ERP.

Actividad 5. Realizar la primera selección: En esta actividad se obtendrá una lista reducida de candida- 
tos (Short list), de 3 a 5 candidatos. A estos candidatos se les aumentará el nivel de exigencia, solicitando reuniones y demostraciones del producto con actividades propias de la empresa, y en caso de que el producto no cuente con alguna función solicitada, se deberá cuestionar acerca de las adecuaciones y desarrollos que se tendrían que hacer al producto. También se deberán organizar visitas a empresas que ya utilizan los ERP seleccionados. Cabe mencionar que durante esta fase se seleccionará el ERP y la casa editora, por lo que es necesario un análisis técnico y económico de la empresa editora.

Actividad 6. Selección final: Basándose en la documentación realizada en las actividades anteriores se toma la decisión final de compra del ERP y se lleva a cabo la negociación con el proveedor ERP. Para complementar esta actividad estamos de acuerdo con uno de los entrevistados, acerca de la necesidad de establecer un contrato formal con el editor, cuidando el costo total, el costo de cada licencia, la forma de pago, etc.

Fase 2. Selección del equipo de consultoría. Una vez seleccionado el producto que se va a implementar el paso siguiente es seleccionar quién lo va a implantar. Esteves y Pastor (2004), Stefanou (2001) y Sternad, Bobek, Dezelak y Lampret (2009) consideran al equipo y consultores ampliamente dedicados como un factor crítico de éxito para proyectos ERP.

Actividad 1. Analizar y documentar necesidades y armar un listado de criterios a evaluar: En varias ocasiones al seleccionar el ERP, se habrá seleccionado ya a la consultoría, ya que el ERP podrá ser implantado solo por el proveedor, por lo que esta fase no será necesaria. Si este no es el caso, se enlistaran los criterios que debe cumplir la empresa consultora para conseguir el proyecto.

Actividad 2. Selección de candidatos: En esta etapa sugerimos llevar a cabo entrevistas a los posibles candidatos sobre una serie de preguntas relacionadas con los procesos actuales y futuros de la organización. Además resulta conveniente solicitar a los candidatos una propuesta de implantación del ERP tomando en cuenta los siguientes aspectos: 1) tiempo estimado de implementación, 2) fecha estimada de arranque del proyecto y de puesta en marcha, 3) costos del proyecto, discriminado el costo de la implantación del costo de soporte post implantación, 4) listado de consultores del equipo de trabajo con los CV de cada uno, 5) plan de contingencia en caso de no cumplir con el tiempo o los costos estimados, 6) alcance del trabajo: implantación, mantenimiento, capacitación a usuario, 7) metodología a utilizar, 8) referencias de otros proyectos en los que han trabajado, 9) listado con las obligaciones y recursos que tendrá que proveer la empresa y 10) experiencia comprobable en la implantación de los módulos que se implantarán en la empresa.

Actividad 3. Selección final: Basándose en la documentación realizada en las actividades anteriores se toma la decisión final de la consultoría que realizará la implantación del ERP y se lleva a cabo la negociación con el proveedor ERP. La cuestión legal de responsabilidades por ambas partes es de trascendental importancia. Por otra parte, durante el periodo de selección de ERP, generalmente es el único momento en el que el consultor se adapta a las necesidades de la empresa e inclusive de la herramienta. Después de haberse seleccionado el ERP y el consultor, los papeles cambian, es decir, la empresa tendrá que adaptarse a la herramienta informática.

Fase 3. Presentación y planificación general del proyecto. Esta fase apunta a presentar el proyecto a las partes involucradas (incluyendo a los utilizadores finales) y a armar un cronograma de implantación.

\section{CONCLUSIÓN}

La fase de selección es transcendental en este tipo de proyectos sociotécnicos, por todo lo que implica a lo largo del proyecto completo de ERP, además de sus implicaciones en la mayor parte del accionar de los departamentos de la empresa. Es importante poder seleccionar el sistema que mejor se adapte a las necesidades de la empresa en varios aspectos, no sólo los económicos sino también los funcionales, estratégicos, técnicos e inherentes al proveedor y su servicio. También se debe encontrar el equilibrio en el producto seleccionado para que el ERP no quede obsoleto al poco tiempo de implantación. Asimismo se debe tomar en cuenta que la complejidad del ERP juega un papel importante en la implantación del sistema. Todos estos puntos hacen pensar que esta etapa es un proceso crítico, no obstante la selección de sistemas ERP no es un área de mucho estudio. Son pocas las metodologías que guíen a los directivos y miembros del equipo de proyecto en este proceso de selección.

Hay diferentes tipos de artículos en el medio académico que van desde propuestas generalizadas para realizar el procesos de selección, hasta propuestas muy puntuales que no contemplan todas las etapas de un proyecto ERP. Nuestro estudio contempla: a) 31 artículos científicos, en donde hay escritos que a su vez estudian 24 artículos más, como el caso de Sternad at al. (2009); b) 4 libros de investigación, en donde se estudiaron varios capítulos; c) 2 tesis de maestría y 2 tesis doctorales, éstas últimas que nos apoyaron para hacer la tabla referente a los análisis microsociológicos. Además, también analizamos artículos pertenecientes a varias disciplinas: ingeniería industrial, informática, sociología, ciencias de las gestión, etc. La diversidad de perspectivas de estos artí- 
culos enriqueció el objetivo de nuestra investigación y se hace necesaria en este tipo de proyectos, debido a la multidisciplinariedad que implica un proyecto tecnológico en una organización empresarial.

Cada una de las áreas en las que dividimos los artículos científicos aportó lo siguiente a nuestro proyecto de investigación:

- Las propuestas metodológicas son estudios generalizados que proveen una guía de pasos o fases, muchas veces probados, que van desde la búsqueda de candidatos hasta la firma del contrato.

- Los casos de estudio ayudan a nuestra investigación a rastrear la aplicación de una metodología de selección específica en el desarrollo de un proyecto ERP.

- Los estudios microsociológicos, por su carácter situacional nos ayudan a puntualizar aspectos muy de lo que sucede a lo largo de la selección de herramientas tecnológicas, tipo ERP.

- Los Factores Críticos de Éxito son un conjunto de recomendaciones para alcanzar un proyecto ERP exitoso, y si bien, no llegan a ser metodologías completas, si aportan aspectos que se podrán considerar para complementar metodologías que involucran a toda la fase de selección.

Estas 4 visiones diferentes de estudiar a los ERP denotan la particularidad de cada uno de los investigadores que llevaron a cabo su respectiva investigación.
Además da una perspectiva de la riqueza científica de donde puede ser abordado el problema de selección de ERP. La riqueza, las diferencias de estudio, las contradicciones y aportaciones similares de varios artículos analizados, nos hace suponer que llevar a cabo la selección de ERP en una empresa, implica una serie de factores, metodologías, análisis y singularidades de donde se va a tener este evento.

Este trabajo nos abre las puertas para conformar una guía (muy abierta y seguramente no lineal) de selección de ERP. Queda pendiente llevar a la prueba esta guía en las Pymes mexicanas retroalimentando las mismas para mejorarlas. Otro estudio que no se llevó a cabo en este artículo, pero que vale la pena hacerlo en próximas investigaciones, correspondería a analizar si los factores de éxito y las metodologías propuestas se pueden generalizar, o más bien, como puntualizamos nosotros, cada una depende del tipo de organización, mercado o cadena de suministro a la que pertenezca la misma, y finalmente al tipo de actores que tienen algo que ver en el proyecto ERP

En la fase 1 de la guía de selección (Actividad 4) destacamos la necesidad de la existencia de una lista de los sistemas ERP que pueden funcionar en las Pymes mexicanas, resaltando las características y recomendaciones de cada ERP, para cada organización. Esta investigación queda pendiente y al mismo tiempo hemos visto que es demandada por las Pymes que buscan adquirir un ERP.

\section{BIBLIOGRAFÍA}

Baptiste, P.; Botta-Genoulaz V.; Niel E. Y Subaï C. (2001): "Du paradigme Suivi / ordonnancement / GPAO au paradigme ERP / APS / MES: révolution ou évolution?", Conception et production Intégrées: CPI'2001, Fès, Maroc.

Chiesa, F. (2004): "Metodología para Selección de Sistemas ERP", Reportes Técnicos en Ingeniería del Software, Vol. $6, \mathrm{n}^{\circ}$ 1, pp. 17-37.

De Pablos Heredero, C. y De Pablos Heredero, M. (2009): Enterprise Information Systems for Business Integration in SMEs: Technological, Organizational and Social Dimensions, Hershey, ed. Business Science Reference.

Estay, C. y Pastor, J. (2002): “Selección de ERP en Pequeñas y Medianas Empresas con un Proyecto de InvestigaciónAcción", 1er. Workshop en: Métodos de Investigación y Fundamentos Filosóficos en Ingeniería del Software y Sistemas de Información.
Esteves, J. y Pastor, J. (1999): “An ERP Lifecycle- based Research Agenda", First International workshop in Enterprise Management and Resource Planning: Methods, Tools and Architectures EMRPS'99, Venice, Italy.

Esteves J. y Pastor J. (2001): “Enterprise Resource Planning Systems Research: An Annotated Bibliography", Communications of the Association for Information Systems (CAIS), Vol. 7, $\mathrm{n}^{\circ}$ 8, August 200.

Esteves, J. y Pastor, J. (2004): “Proyectos ERP exitosos como base de ventajas competitivas". Empresa, n 9, pp. 32-44.

Expósito, M.; Tomás, J. y Vicedo, J. (2004): Metodología para el análisis del sistema de información de una empresa de fabricación de tejidos y propuesta de una solución, VIII Congreso de Ingeniería de Organización Leganés, España.

Forger, G. (2000): "ERP goes mid-market", Modern Materials Handling, Vol. 55, $\mathrm{n}^{\circ}$ 1, Leganés, España, pp.65-71.
García, A., (2008): “Estudio para la implantación de un ERP en una empresa textil", Tesis de Maestría en Ingeniería de la Universidad Autónoma de Barcelona.

Rivera, I, (2005): “La selección de un ERP. Dinámicas colectivas y procesos de aprendizaje de los actores", These de docteur de l'Institut National Polytechnique de Grenoble, Francia.

Segrestin, D. (2004): Le mythe de l'organisation intégrée. Les progiciels de gestión, Sciences de la Société, $\mathrm{n}^{\circ} 61$, pp. 3-15.

Sheu, C.; Chae, B. y Yang, C. (2004): "National differences and ERP implementation: issues and challenges", Innsbruck, Omega, Vol. 32, $\mathrm{n}^{\circ} 5$.

Stefanou, C. (2000): "The Selection Process of Enterprise Resource Planning ERP Systems", Proceedings of AMCIS 2000, Long Beach, pp. 989-990 
Stefanou, C. (2001): "A framework for the ex-ante evaluation of ERP software", European Journal of Information Systems, pp. 204- 215.

Sternad, S.; Bobek, S.; Dezelak, Z. y Lampret, A. (2009): Enterprise Information Systems for Business Integration in SMEs: Technological, Organizational and Social Dimensions, Hershey, ed. Business Science Reference, pp. 412-431.
Teltumbde, A. (2000): “A framework of evaluating ERP projects", International Journal of Production Research, $\mathrm{n}^{\circ} 38$, pp. 4507-4520.

Urcelay, A., (2003): "Impacto de los sistemas ERP en la Gestión Logística de las empresas manufactureras. El Caso de la Comunidad Autónoma del País Vasco", Tesis doctoral de la Universidad de Deusto, San Sebastián, España.
Verville J., H.A. (2002): "A six-stage model of the buying process for ERP software", Science Direct, p. 10.

Wei, C.; Chien, C. y Wang, M. (2005): “An AHP-based approach to ERP system selection", Intern. Journal of Production Economics, Vol. 96, n 1, pp. 47-62. 